\title{
Heinrich Gerlachs Dokumentarromane Durchbruch bei Stalingrad (1945/2016) und Odyssee in Rot (1966/2017) und Aspekte der zeitgenössischen Rezeption
}

\section{Einleitung}

Heinrich Gerlach ist in der deutschen Öffentlichkeit seit 2016 wieder ein Begriff. Die Publikation seines bis dahin verschwunden geglaubten Romans Durchbruch bei Stalingrad (1945/2016) fand eine umfangreiche Rezeption, und innerhalb einer kurzen Zeit ergaben sich Übersetzungen ins Holländische, Französische und Englische. Auch die Rechte für eine Übersetzung ins Russische wurden vergeben. ${ }^{1}$

Die breite Resonanz, die der Roman erfuhr, machte den in den letzten Jahrzehnten vergessenen Autor und Zeitzeugen wieder bekannt. Das Buch hatte Heinrich Gerlach in sowjetischer Kriegsgefangenschaft im Sommer 1943, ein halbes Jahr nach der Katastrophe, begonnen, und mit dem Kriegsende am 8. Mai 1945 setzte er den Schlusspunkt. Danach war der Autor in verschiedenen Kriegsgefangenenlagern; 1949 wurde das Romanmanuskript vom sowjetischen Geheimdienst konfisziert und war bis zu seiner nach jahrzehntelanger Recherche erfolgten Wiederauffindung in einem russischen Militärarchiv verschlossen. ${ }^{2}$ Dieser Sta-

1 Siehe Gerlach, Heinrich. Doorbraak bij Stalingrad. Hg. Carsten Gansel. Amsterdam: Uitgegeven door Xander Uitgevers BV 2017; Gerlach, Heinrich. Éclairs lointains Percée Stalingrad. Hg. Carsten Gansel. Paris: Éditions Anne Carrière 2017; Gerlach, Heinrich. Breakout at Stalingrad. Hg. Carsten Gansel. London: Apollo/Head of Zeus Ltd. 2018.

2 Das Urmanuskript wurde im Februar 2012 vom Verfasser in einem russischen Archiv wiedergefunden und in einem aufwendigen Prozess zur Edition beim Galiani Verlag (Berlin) gebracht. Vgl. ausführlich Gansel, Carsten. „Nach 70 Jahren aus der Kriegsgefangenschaft zurück - Heinrich Gerlachs Roman Durchbruch bei Stalingrad und seine abenteuerliche Geschichte“. In: Heinrich Gerlach. Durchbruch bei Stalingrad. Hg. Carsten Gansel. Berlin: Galiani 2016, 519-693. 2017 wurde dann Gerlachs zweiter Roman, Odyssee in Rot, ebenfalls bei Galiani mit einem umfangreichen Nachwort ediert. Vgl. Gansel, Carsten. „Widerstandsheld, Vaterlandsverräter, wacher Demokrat und Zeitzeuge? - Heinrich Gerlach, seine Odyssee durch die sowjetischen Gefangenenlager und sein Schicksal in der sich neu formierenden Bundesrepublik“. In: Heinrich Gerlach. Odyssee in Rot. Bericht einer Irrfahrt. Hg. Carsten Gansel. Berlin: Galiani 2017, 691-915. Die nachfolgende Darstellung hat ihre Grundlage in den beiden Editionen sowie den Nachworten. 
lingradroman - an anderer Stelle wurde dessen höchst ungewöhnliche Entstehungs- und Veröffentlichungsgeschichte ausführlich beschrieben - ist aber nicht das einzige Buch, das Heinrich Gerlach veröffentlicht hat. Sein zweiter Roman, Odyssee in Rot. Bericht einer Irrfahrt (1966/2017), ist zwar unter weniger existenziellen Umständen entstanden, schildert aber ebenfalls dramatische Ereignisse. Einmal mehr zeigt sich Heinrich Gerlach als Ausnahmefigur in der deutschen Literaturlandschaft nach 1945. Und dies hat nicht zuletzt damit zu tun, in welcher Weise der Autor dokumentarisches Material nutzt. Odyssee in Rot schildert das Schicksal deutscher Soldaten und Offiziere nach dem Ende der Kriegshandlungen im Mai 1945 und ihre langjährige Gefangenschaft bis zur Rückkehr nach Deutschland. Der Dokumentarroman endet mit dem Eintreffen des Kriegsgefangenen Breuer - dies ist das alter ego von Heinrich Gerlach - in Berlin. Es finden sich keine Aussagen darüber, wie es mit Gerlach und den anderen kriegsgefangenen deutschen Offizieren nach der Rückkehr im Nachkriegsdeutschland weitergeht. Der Bericht schließt mit einer emotional bewegenden Episode, in der Breuer alias Heinrich Gerlach am 22. April 1950 nach fast 11 Jahren endlich seine Frau und die Kinder wiedersieht:

Langsam stieg er die Treppe hinauf zur Sperre. Auf der Schulter den Luftwaffenrucksack [...]. Die Beine wurden ihm schwerer und schwerer. Da war die Sperre. Darüber die große Bahnhofsuhr. Und dahinter, in den Winkel an den Schaltern gedrückt wie in Furcht, eine Frau... Breuer ging auf die Frau zu. Ein Junge stand neben ihr, so groß wie sie selbst. Eine Kinderzeichnung, Baum und Haus, und zwei gelbe Sonnen darüber. Zwei Sonnen, die einen Bunker von Stalingrad erhellten... „Er wird eingesegnet“, sagte Irmgard und drückte den Jungen an sich. „Morgen wird er doch eingesegnet!“ Und nun weinte sie. Es war 23 Uhr 04 $\ldots^{3}$

Heinrich Gerlachs Tochter, Dorothee Wagner, hat später bestätigt, dass sich das Geschehen bei der Rückkehr des Vaters genauso abgespielt hat. ${ }^{4}$ Es geht also in beiden Texten um ein besonderes Verhältnis von Fakt und Fiktion. Im Nachwort zu seinem Roman Durchbruch bei Stalingrad begründet Heinrich Gerlach den fiktionalen, also künstlerischen Charakter seiner Darstellung wie folgt:

Dieses Buch ist ein Roman. Hinter den Trägern der Handlung (soweit sie nicht - wie Feldmarschall Paulus oder General v. Seydlitz - einer breiteren Öffentlichkeit bekannt geworden sind) bestimmte Gestalten der Wirklichkeit zu suchen, ist abwegig und könnte zu einem Un-

3 Gerlach, Heinrich. Odyssee in Rot. Bericht einer Irrfahrt. Berlin: Galiani 2017, 687.

4 Gansel, Carsten: Gespräch mit Dorothee Wagner und Ingeborg Gerlach, der Schwiegertochter von Heinrich Gerlach, im August 2012. Ich danke Dorothee Wagner, die 2014 verstorben ist, und Ingeborg Gerlach ausdrücklich für die Bereitschaft zu dem Gespräch und die Genehmigung, die Informationen zu nutzen. 
recht gegen Tote und Lebende werden. Dennoch ist in diesem Buch nichts ,erfunden'. Alles, was die Romanhandlung an Begebenheiten schildert, war irgendwann und irgendwo auf den Schneefeldern vor Stalingrad und in den Trümmern der Stadt einmal Wirklichkeit. Nur mit Ort, Zeit und den beteiligten Personen ist der Verfasser nach eigenem Ermessen verfahren. ${ }^{5}$

In der Taschenbuchausgabe, die 1962 erschien, finden sich im Nachwort sodann Ergänzungen, die die Darstellungsweise betreffen. Heinrich Gerlach macht darauf aufmerksam, dass das Buch ,trotz der romanhaften Gestaltung Anspruch auf dokumentarischen Wert im gegenständlichen und seelischen Bereich erhebt" . Dass die Figur des Oberleutnants Breuer als alter ego von Heinrich Gerlach zu lesen ist, hat der Autor selbst wiederholt betont. Ein wenig anders stellt Gerlach das Verhältnis zwischen Fakt und Fiktion in der Odyssee in Rot von 1966 dar. „Dieses Buch ist ein Bericht“, notiert er in der Vorbemerkung:

Die eingearbeiteten Reden, Zeitungsartikel, Aufrufe, Rundfunkvorträge und Predigten sind echt und dokumentarisch zu belegen. [...] Die handelnden Personen erscheinen vorwiegend unter ihrem eigenen Namen, einige der noch Lebenden wurden umbenannt, zumeist auf ihren Wunsch. Wenige wurden ganz oder teilweise erfunden. ${ }^{7}$

Noch stärker, als es bereits in Die verratene Armee (1957) und dem wiederentdeckten Original Durchbruch bei Stalingrad der Fall ist, existiert also so etwas wie ein „autobiographischer Pakt“ zwischen Autor und Leser. Die Hauptfigur und der Autor sind nicht nur eng aneinandergerückt, wie dies bei vielen Autoren der Fall ist, sondern nahezu identisch. Die Leser können daher eine weitgehende Übereinstimmung zwischen der Hauptfigur, dem Oberleutnant Richard Breuer, und Heinrich Gerlach annehmen. Insofern handelt es sich um die Geschichte einer „tatsächlichen Person über ihre eigene Existenz“, es geht mithin um das ,persönliche Leben“ von Heinrich Gerlach und seinen Weg durch die Gefangenschaft. ${ }^{8}$ Anders als in einem Roman, bei dem die erzählte Geschichte von ,nicht-wirklichen Ereignissen, nicht-wirklichen Figuren, nicht-wirklichen Orten oder nichtwirklichen Zeiten“9 handelt, geht es bei Heinrich Gerlach genau um das Gegen-

5 Gerlach, Heinrich. Durchbruch bei Stalingrad. Hg. Carsten Gansel. Berlin: Galiani 2016, 515.

6 Gerlach, Heinrich. „Nachwort“. In: Heinrich Gerlach. Die verratene Armee. Ein StalingradRoman. 2., bearbeitete Taschenbuchausgabe. München: Wilhelm Heyne 1976, 309-311, hier 309. 7 Gerlach: Odyssee in Rot, 5.

8 Lejeune, Philippe. Der autobiographische Pakt. Aus dem Französischen von Wolfram Bayer und Dieter Hornig. Frankfurt a. M.: Suhrkamp 1994, 14.

9 Zipfel, Frank. „Autofiktion. Zwischen Grenzen von Faktualität, Fiktionalität und Literarizität“. In: Revisionen. Grundbegriffe der Literaturtheorie. Hg. Fotis Jannidis u. a. Berlin/New York: De Gruyter 2009, 285-314, hier 290. 
teil: Alle Figuren und Ereignisse sind verbürgt. Tagebuchnotizen von Heinrich Gerlach, die nach der Fertigstellung der Edition von Durchbruch bei Stalingrad aufgefunden wurden, sowie Dokumente aus russischen Archiven belegen sämtliche Stationen der Gefangenschaft, wie sie in der Odyssee in Rot geschildert werden. Auch die Schlüsselepisoden, etwa bei der Gründung des Bundes Deutscher Offiziere (BDO) vom September 1943, beruhen auf den Erlebnissen von Heinrich Gerlach und sind durch die historischen Fakten belegt. Gerlach baut entsprechend in den Roman Dokumente aus der Gründungsveranstaltung ein. Es ist eben dieser Aspekt, der in dem hier in Rede stehenden Rahmen dazu führt, von einem Dokumentarroman zu sprechen. Insofern bietet sich an dieser Stelle wenngleich es um einen gänzlich anderen Gegenstand geht - ein Vergleich mit Ursula Krechels Roman Landgericht an, der 2012 mit dem Deutschen Buchpreis ausgezeichnet wurde und einmal mehr eine Diskussion um das Verhältnis von fictional und factual auslöste. ${ }^{10}$ Andreas Platthaus hat in seiner Besprechung auf den dokumentarischen Kern des Romans verwiesen und notiert: „Kornitzer hat wirklich gelebt, er hieß anders, doch seinen Fall hat Ursula Krechel aus den Akten des Landesarchivs von Rheinland-Pfalz akribisch rekonstruiert. Akten aber sind unpersönlich, deshalb hat sie einen Roman geschrieben." Würde man allerdings Heinrich Gerlachs Roman hinsichtlich des Einsatzes von dokumentarischem Material mit jenem von Ursula Krechel vergleichen, dann stellten sich deutliche Unterschiede in quantitativer Hinsicht heraus. Anders gesagt, der Umfang, in dem Gerlach Dokumente einbaut und Figuren der Zeitgeschichte zu Wort kommen lässt, ist deutlich umfassender als bei Ursula Krechel. Gleichwohl kann man beide Texte - orientierte man sich an neueren Diskussionen - als Dokufiktionen bezeichnen. ${ }^{12}$ Offensichtlich wird aber auch, dass einzig die konkrete analytische Arbeit am Text, mithin die narratologische Analyse, die Frage nach dem jeweils existierenden Verhältnis von Fiktionalität und Faktualität beantworten kann.

\footnotetext{
10 Siehe dazu meinen Beitrag: Gansel, Carsten. „Ursula Krechels Landgericht (2012) und die ,verlorenen Erinnerungen' der Nachkriegszeit oder Erinnerungskulturen zwischen Verdrängen und Vergessen“. In: Deutschunterricht (6/2019), 32-43; vgl. außerdem den Beitrag von Eva-Maria Konrad in diesem Band.

11 Platthaus, Andreas. „In der Sache Kornitzer“. In: Frankfurter Allgemeine Zeitung vom 10. Oktober 2012.

12 Bidmon, Agnes. „Dokufiktion - Neue Formen realistischen Erzählens in der deutschsprachigen Gegenwartsliteratur“. In: Dokumentarisches Erzählen - Erzählen mit Dokumenten in Literatur, Theater und Film. Hg. Carsten Gansel, Peter Braun. Berlin: Okapi Verlag 2020, 103-130.
} 


\section{Heinrich Gerlach und das Dokumentarische in Durchbruch bei Stalingrad}

Nachfolgend sei ausgesprochen selektiv der Versuch gemacht, den Umgang des Autors mit dem Dokumentarischen anzudeuten. Der von Gerlach bereits zu Beginn des Romans Durchbruch bei Stalingrad eingeführte Hauptmann Engelhard sieht sich im Bunker mit der aussichtslosen Situation der 6. Armee konfrontiert:

Engelhard hörte ernst und gesammelt zu. Zuweilen blickte er Zuspruch heischend in die dunkle Ecke. Doch dort blieb alles still. „Ja“, sagte er schließlich, „das ist die Auflösung. Ich glaube, wir sind am Ende. Es bleibt nichts anderes, als so anständig wie möglich von der Bildfläche abzutreten.“ „Anständig?“ lachte Major Siebel ungeniert. „Schöner Anstand! Sollen die Herren von der Armee sich doch mal rausrühren aus ihren Löchern! Soll Paulus doch mal rausgehen auf die Straßen und sehen, ob das noch anständig sterben heißt, was sich da abspielt... Bildet sich immer noch ein, daß er Soldaten hat, daß er führen kann, Verteidigungslinien aufbauen kann! Soll er doch mal rausgehen! So etwas war in der ganzen Weltgeschichte noch nicht da!“13

Die Figurenrede des Majors Siebel entspricht durchaus den Auffassungen von Heinrich Gerlach, der in seinen Beiträgen in der Zeitung Freies Deutschland die Rolle von Generalfeldmarschall Paulus ausgesprochen kritisch bewertet. ${ }^{14} \mathrm{Ganz}$ anders sieht er General Walther von Seydlitz, mit dem er nach dessen Entlassung aus der Gefangenschaft über Jahre in einem engen Kontakt stehen wird. ${ }^{15}$ Sofort nach der Rückkehr des ehemaligen Generals nimmt Heinrich Gerlach - das Tagebuch belegt es - Kontakt zu ihm auf. Im erst 2016 zugänglich gewordenen Nachlass von Walther von Seydlitz-Kurzbach fanden sich schließlich Teile des Briefwechsels zwischen Gerlach und von Seydlitz, die Gerlachs Vorgehen illus-

13 Gerlach: Durchbruch bei Stalingrad, 353.

14 Vgl. Gerlach, Heinrich. „Wendepunkt Stalingrad. Rückzug nach Osten“. In: Freies Deutschland vom 21.11.1943, 1; Gerlach, Heinrich. „Der ,Geist von Stalingrad““. In: Freies Deutschland vom 26.03.1944, S. 1; Gerlach, Heinrich. „2 Jahre nach Stalingrad. Der neue Weg“. In: Freies Deutschland vom 04.02.1945, S. 1. Birgit Petrick hat sich in einer verdienstvollen Arbeit mit der Zeitung Freies Deutschland auseinandergesetzt und dabei auch eine akribische Auflistung aller Beiträger vorgenommen. Siehe Petrick, Birgit. ,Freies Deutschland“ - die Zeitung des Nationalkomitees ,Freies Deutschland‘ (1943-1945). München: K. G. Saur 1979, 296-351.

15 Gerlach und Walther von Seydlitz, die in der Folgezeit engen Kontakt halten, werden sich dann über die Entstehung der Odyssee in Rot entzweien. Es geht dabei um die dokumentarischen Details der Gründung des Bundes Deutscher Offiziere (BDO). 
trieren. ${ }^{16}$ Die Briefe belegen das ausgesprochen vertrauensvolle Verhältnis, das zwischen beiden nach der Rückkehr des Generals aus der Kriegsgefangenschaft im Oktober 1955 entsteht. In der Folgezeit kommt es zu einem engen Austausch zwischen Heinrich Gerlach und dem 24 Jahre älteren Walther von Seydlitz, der sich auf die Fakten um die 6. Armee im Winter 1942/43 bezieht. Da der ehemalige General die Entstehung des Urmanuskripts im Lager Lunjowo (1943-1945) mitverfolgt und unterstützt hat, nimmt es nicht wunder, wenn Gerlach wenige Wochen, nachdem er den Verlagsvertrag mit der Nymphenburger Verlagshandlung abgeschlossen hat, Walther von Seydlitz diese glückliche Entwicklung nicht nur umgehend mitteilt, sondern ihm auch das Manuskript am 20. November 1956 mit folgenden einleitenden Worten zuschickt:

Zu meiner großen Freude ist es mir gelungen, mein Stalingradbuch unterzubringen und damit ein langes und schwieriges Bemühen endlich zu einem glücklichen Abschluß zu bringen. Der Verlag, der angebissen hat, ist die ,Nymphenburger Verlagshandlung، in München. Dort wird das Buch im nächsten Herbst erscheinen, voraussichtlich unter dem Titel „Wie das Gesetz befahl“. Der Verlag drängt sehr, das Buch möglichst bald druckfertig zu erhalten. ${ }^{17}$

Der Brief ist im Hinblick auf die Rolle des Dokumentarischen in mehrfacher Hinsicht aufschlussreich. Einerseits findet sich der Hinweis auf den ursprünglich ins Auge gefassten Titel „Wie das Gesetz befahl“, andererseits wird belegt, dass offensichtlich das gesamte Manuskript an Walther von Seydlitz geht. Dabei kommt es dem Autor Gerlach nicht darauf an, dass von Seydlitz grundlegende Einlassungen macht und als Korrektor fungiert. Ihm ist klar, und dies drückt er auch unmissverständlich gegenüber von Seydlitz aus, dass dieser gegebenenfalls manches zur Katastrophe von Stalingrad anders sehen wird. Dies umso mehr, da es sich um keine wissenschaftliche Arbeit, sondern um einen Roman handeln soll:

Wenn ich Ihnen deshalb heute das Manuskript zur Durchsicht zusende, so nicht, um Ihnen damit etwas Neues zu bieten. Sie kennen das Buch in seiner 1. Fassung von Lunjowo her und auch seine Tendenz, die nicht geändert, sondern höchstens noch vertieft wurde. Und ich glaube, daß Sie damals dem Buch - gerade auch in Hinblick auf Plievier - mit einer Sympathie gegenüberstanden. Natürlich wird es bei einem so vielschichtigen Thema wie Stalingrad nicht ausbleiben, daß sie vieles anders sehen müssen und gewertet wissen wollen als ich. Das wird man in Kauf nehmen müssen, und das Buch soll ja auch nichts vorwegnehmen, was Sie selbst zu diesem Thema zu sagen haben werden. Es ist also nicht

16 Der Briefwechsel zwischen Heinrich Gerlach und Walther von Seydlitz ist nicht vollständig. Von Heinrich Gerlach finden sich lediglich drei Briefe, die allerdings einen präzisen Einblick in die Verständigung zwischen ihm und von Seydlitz geben.

17 Heinrich Gerlach an Walther von Seydlitz, 20.11.1956. In: Nachlass N55: Walther von SeydlitzKurzbach. Bundesarchiv, Militärarchiv Freiburg, Signatur N55/121, Blatt 9. 
meine Absicht, Sie um eine so subtile Kritik zum einzelnen zu bitten, wie sie bei dem Buch von Scheurig nötig war. Dieses hier ist keine wissenschaftliche Untersuchung, sondern ein Roman mit allen den Freiheiten, die man dieser Gattung nun einmal zugestehen muß. Das kurze Vorwort zeigt ja, wie das Geschilderte verstanden werden will. ${ }^{18}$

Gerlach geht es schlichtweg darum, dass der General einige historische Fakten prüft, die sein Agieren in Stalingrad betreffen. Nachfolgend benennt er daher ausgewählte Stellen im Manuskript, zu denen er die Meinung des Generals von Seydlitz hören möchte. Entsprechend heißt es erläuternd:

Worauf es mir vor allem ankommt, ist folgendes: Sie selbst sind eine der wenigen Gestalten, die in dem Buch unter ihrem wirklichen Namen erscheinen. Es ist daher für mich ein selbstverständliches Gebot der Loyalität, Ihnen vor allem diese drei oder vier Stellen (S. 84 ff., 94, 358 ff., 456) vorzulegen mit der Bitte zu überprüfen, ob diese Stellen Ihre Billigung soweit finden, daß Sie mit der Verwendung Ihres Namens einverstanden sein können. Insbesondere gilt das für die Szene S. 84 ff., die ich Ihnen schon damals in Lunjowo vorgelesen habe und in die ich inzwischen einige kurze, aber wichtige Stellen aus ihrer Denkschrift im Wortlaut eingearbeitet habe. ${ }^{19}$

In der Episode, die Gerlach wichtig ist, wird die Person bzw. Figur des Generals Walther von Seydlitz eingeführt. In einem Bunker der Führungsabteilung der 6. Stalingradarmee tappen Schritte die Stiege herunter und eine schneebestäubte Gestalt schiebt sich in den Raum, der General der Artillerie von Seydlitz. Nach einem knappen Dialog setzt der Erzähler mit einer Charakterisierung der Person ein, die die Herkunft und das Äußere der Figur mit Sympathie erfasst:

Der General lacht bitter auf, während er sich aus seinen Umhüllungen schält. Er ist ein Nachfahre des friderizianischen Reitergenerals, und auch er selbst ist ein passionierter Reiter. Das verrät die trotz des fast weißen Haares noch jugendlich straffe Gestalt, und auch das scharf geprägte Gesicht, jetzt zorngerötet, hat etwas von einem Rassepferd. ${ }^{20}$

Im Weiteren geht es um eine für den Verlauf der Stalingrad-Katastrophe entscheidende Situation: Auf einen Bericht des Oberbefehlshabers der 6. Armee, General Paulus, in dem dieser die aussichtslose Lage schildert und den Ausbruch aus dem

18 Gerlach an von Seydlitz, 20.11.1956.

19 Gerlach an von Seydlitz, 20.11.1956. (Unterstreichung i. O.).

20 Gerlach, Heinrich. Die verratene Armee. München: Nymphenburger Verlagshandlung 1957, 96. In der Urfassung heißt es dagegen: „Er nahm die Feldmütze vom Kopf und strich sich mit der Hand über das weiße, an den Schläfen kurz geschnittene Haar. Dann legte er seine Tarnjacke ab, die das Ritterkreuz mit dem Eichenlaub freigab. Seinem faltigen Gesicht nach mochte der General um die Fünfzig herum sein, die straffe Reitergestalt, Ausdruck einer verpflichtenden Familientradition, ließ ihn erheblich jünger erscheinen“ (Gerlach: Durchbruch bei Stalingrad, 96-97). 
Kessel nahelegt, ist aus dem Führerhauptquartier - wie es im Roman heißt - der lakonische Bescheid gekommen: „Einigelung beider Armeen unter Paulus’ Oberbefehl“. Oberst Clausius ist schockiert und General von Seydlitz außer sich. Entsprechend legt Gerlach ihm folgende Worte in den Mund:

Ein Wahnsinn ist es, eine ausgemachte Idiotie! Zwei ganze Armeen lassen sich freiwillig einkesseln, wo jetzt wahrscheinlich ein Stoß genügen würde, um hier herauszukommen. Sowas gab’s in der ganzen Weltgeschichte noch nicht! ${ }^{21}$

Der General kann sich mit dem Einigeln im Kessel nicht abfinden, und gemeinsam mit Oberst Clausius macht er sich daran, einen schriftlichen Protest auf Grundlage einer klaren Lagebeurteilung abzufassen. Heinrich Gerlach baut nun in seinen epischen Text aus der 1956 zur Verfügung stehenden Denkschrift des Generals von Seydlitz, die den Titel Die Beurteilung der Lage der 6. Armee im Kessel von Stalingrad trägt, in dokumentarischer Manier maßgebliche Teile ein. Es sind dies vor allem jene Passagen, die den Kern der von Seydlitz'schen Argumentation vom 25. November 1943 ausmachen. So kann man in der neu entstandenen Fassung mit dem späteren Titel Die verratene Armee lesen:

„Es ergibt sich unwiderleglich“, diktiert der General; „die Armee kann bei Verharren in der Igelstellung nur dann der Vernichtung entgehen, wenn in wenigen Tagen Entsatz wirksam wird, so daß der Feind seine Angriffe einstellen muß. Hierfür liegen keinerlei Anzeichen vor..."

Gerlach hält sich bei der Darstellung nahezu wörtlich an die Ausarbeitung des Generals vom November 1943.22 Klar und deutlich lässt er von Seydlitz im Roman das formulieren, was dieser in der Realität des Kessels von Stalingrad als kommandierender General des LI. Armeekorps an den Oberbefehlshaber, Generalfeldmarschall Paulus, ohne Beschönigung geschrieben hatte:

21 Gerlach: Die verratene Armee. 1957, 97. In der Urfassung fällt die Wertung noch weitaus schärfer aus, denn es heißt: „Ein hirnverbrannter Wahnsinn! Die freiwillige Einigelung von 22 Divisionen, wo hat es so was jemals gegeben! Das bekommt doch nur ein Verrückter fertig!“ (Gerlach: Durchbruch bei Stalingrad, 96-97) Da Gerlach im Gefangenenlager allerdings nicht über das Dokument selbst verfügt, also über den Lagebericht vom 25. November 1943, muss er die Argumentation umschreiben und kann nicht den getreuen Wortlaut wiedergeben.

22 Vgl. Seydlitz, General Walther von. „Die Beurteilung der Lage der 6. Armee im Kessel von Stalingrad“. In: Hans Martens. General von Seydlitz 1942-1945. Analyse eines Konflikts. Berlin: edition kloeden 1971, 76-81. In dem Dokument, aus dem Gerlach zitiert, heißt es: „Operativ ergibt sich daher unwiderleglich: die 6. Armee kann bei Verharren in der Igelstellung nur dann der Vernichtung entgehen, wenn der Entsatz in wenigen, d.h. 5 Tagen, soweit wirksam wird, daß der Feind seine Angriffe einstellen muß. Hierfür liegt nicht ein einziges Anzeichen vor“. Seydlitz: „Die Beurteilung der Lage der 6. Armee im Kessel von Stalingrad“, 79. 
Der Befehl, den Igel zu halten, bis Hilfe heran ist, hat keinerlei reale Grundlagen. Er ist unausführbar und muß zwangsläufig den Untergang der Armee zur Folge haben. Wenn das vermieden werden soll, so muß unweigerlich ein anderer Befehl herbeigeführt werden... ${ }^{23}$

Die Beurteilung der Lage beruht - das stellt von Seydlitz in der ihm eigenen Geradlinigkeit heraus - ,auf nüchterner Erkenntnis der tatsächlichen Gegebenheiten“24. Sollte es keinen anderen Befehl geben, so fordert der General vom Oberbefehlshaber Paulus, dann müsse man sich „die Handlungsfreiheit selbst nehmen“25. Auf den Einwand von Oberst Clausius im Roman, dass dies Meuterei sei und unter Umständen den Kopf koste, lässt Gerlach Walther von Seydlitz kurz nachdenken und dann ruhig sagen:

„Sie mögen Recht haben, Clausius. Aber darum geht es jetzt nicht. Es geht nicht um mich und um Sie und um Paulus oder sonst irgendeinen, der hier Verantwortung trägt... Es geht um dreihunderttausend deutsche Soldaten."26

Heinrich Gerlachs Wertschätzung des Generals Walther von Seydlitz entspringt dem Wissen, dass hier ein Offizier von sich selbst absieht, dem „eigenen Gewissen gegenüber der Armee und dem deutschen Volk“ folgt und gegen einen Befehl, der den Tod von Hunderttausenden bedeuten kann, protestiert. In seinem Brief vom 20. November 1956 setzt Gerlach dem General auseinander, dass es sich bei der vorliegenden Episode um eine Mischung von Fakt und Fiktion handelt und im Interesse des Romans daher nicht jedes Detail mit der Realität vom November 1943 übereinstimmen muss:

Es geht nun nicht darum, ob das Gespräch mit Clausius und die Entstehung der Denkschrift sich wirklich genauso abgespielt haben. Natürlich war es nicht so! Clausius war ja, wie Sie letztlich erzählten, bei Paulus mit dabei. Außerdem ist bei mir die Abfassung der Denkschrift aus kompositorischen Gründen um einen Tag vorverlegt, was gewisse Akzentverschiebungen notwendig gemacht hat. ${ }^{27}$

23 Gerlach: Die verratene Armee. 1957, 99.

24 Seydlitz: „Die Beurteilung der Lage der 6. Armee im Kessel von Stalingrad“, 76.

25 Gerlach: Die verratene Armee. 1957, 100. In der Denkschrift heißt es im vollen Wortlaut: „Hebt das OKH [das Oberkommando des Heeres] den Befehl zum Ausharren in der Igelstellung nicht unverzüglich auf, so ergibt sich vor dem eigenen Gewissen gegenüber der Armee und dem deutschen Volk die gebieterische Pflicht, sich die durch den bisherigen Befehl verhinderte Handlungsfreiheit selbst zu nehmen und von der heute noch vorhandenen Möglichkeit, die Katastrophe durch eigenen Angriff zu vermeiden, Gebrauch zu machen“. Seydlitz: „Die Beurteilung der Lage der 6. Armee im Kessel von Stalingrad“, 81.

26 Gerlach: Die verratene Armee. 1957, 100 (Auslassungszeichen i. O.).

27 Gerlach an von Seydlitz, 20.11.1956. 
Gerlach verweist einmal mehr auf den künstlerischen Charakter der Darstellung und fasst seine Intention in einer Weise zusammen, die dem General anschaulich machen soll, wie die Verwandlung von Leben in Literatur funktioniert und worauf es seiner Meinung nach ankommt:

Es geht also hier nicht um die „Wahrheit“ in solchen Einzelheiten, sondern um die höhere Wahrheit, um die Aufhellung der Hintergründe, um die Zwangslage, die seelische Not, die Urtiefen der Persönlichkeit, die zu diesem ersten Aufbegehren gegen Hitler geführt haben und führen mußten. Ähnliches gilt für die zweite große Szene (S. 358 ff.). Ich weiß, daß Jaenicke damals noch dabei war (vielleicht kann man das übrigens noch ändern) und daß die Einzelheiten sich so nicht abgespielt haben. Aber auch hier geht es ja nicht darum, sondern um die Aufhellung der Persönlichkeiten, ihres Denkens und Wollens. Das Buch ist eben kein Tatsachenbericht, sondern ein Roman..$^{28}$

Explizit stellt Heinrich Gerlach noch einmal das Grundprinzip seines Ansatzes heraus, wenn er sich an solchen Stellen vom Tatsachenbericht absetzt und auf das Romanhafte verweist. Allerdings darf die künstlerische Freiheit aus seiner Sicht nicht zur Verfälschung der Realität von Stalingrad führen und schon gar nicht die Rolle von realen Personen umdeuten, etwa jener des Generals von Seydlitz. In seinem Schreiben begründet er, warum ihm gerade diese beiden Episoden, in denen der General vorkommt, so wichtig sind. „Warum nun überhaupt diese beiden Szenen? Warum Ihr Name“, fragt er und gibt umgehend die Antwort:

Ich will damit Ihre historische Rolle bei Stalingrad deutlich machen. Ich will zeigen, daß Sie als einziger damals die Situation mit allen ihren weitreichenden Konsequenzen durchschauten und zum Handeln aufriefen, ohne freilich nach Lage der Dinge die Möglichkeit zu haben, diese Konsequenzen für sich selbst bis ins letzte hin zu ziehen. Der Leser muß begreifen, wer dieser General von S. ist, was er damals erkannte und tat und was er noch nicht tun konnte. Alles dies soll in ihm das Verständnis wecken für das, was dann in der Gefangenschaft folgte. So glaube ich eine Bresche zu schlagen in die allgemeine Verständnislosigkeit gegenüber der „Beweg. F.D:“ und ein wenig die psychologische Basis vorzubereiten für das, was Sie selbst eines Tages zu alledem sagen werden. ${ }^{29}$

Was Heinrich Gerlach in diesem Brief formuliert, ist insofern zukunftsweisend, als er die Leistung des Generals Walther von Seydlitz im Kessel von Stalingrad und in der Gefangenschaft würdigt, ganz entgegen dem vorherrschenden Unverständnis, der Feindseligkeit und der Verfemung, auf die dieser zunächst in der Bundesrepublik trifft, als er als einer der letzten 1955 aus der sowjetischen Kriegsgefangenschaft in die Bundesrepublik zurückkehrt. Gerlach gelangt damit schon Mitte

28 Gerlach an von Seydlitz, 20.11.1956. (Herv. i. O.)

29 Gerlach an von Seydlitz, 20.11.1956. 
der 1950er Jahre zu einer Einschätzung der historischen Bedeutung des Walther von Seydlitz, wie sie sich erst in einem langen Prozess von den 1970er bis in die 1990er (!) Jahre durchsetzen wird. Offensichtlich ist freilich auch, dass der Verratenen Armee - im Unterschied zur Urfassung Durchbruch bei Stalingrad - der Diskurs der 1950er Jahre eingeschrieben ist, denn Gerlach spricht nicht zufällig davon, dass erst einmal eine „psychologische Basis“ für eine vorurteilsfreie Betrachtung des BDO und jener hohen Offiziere zu schaffen ist, die in der Gefangenschaft Widerstand gegen Hitler geleistet haben. „Die heutige psychologische Situation erfordert es, das mit aller Vorsicht zu tun“30, heißt es mit Blick auf die damalige Situation.

Walther von Seydlitz antwortet drei Wochen später in seiner heute nur schwer lesbaren spezifischen Variante einer Sütterlinschrift mit einem Plädoyer für den Roman, an dem er die dokumentarische Qualität hervorhebt:

Lieber Gerlach! Gestern Abend wurde ich fertig mit der mehrfach erschütternden Lektüre Ihres Buches. Meine Stellungnahme - in gekürzter Form - die Kürze zwingt dazu.

1) Allgemein: Es ist Ihnen m. E. sehr gut gelungen, das Inferno dieses ganzen Wahnsinns bis in die letzten höllischen Tiefen vorzustellen. Tiefer geht's nimmer und das ganze Grauen, das einen schon sehr bald umfängt bei der Lektüre des Buches läßt einen bis zum Schluß nicht mehr los. So muß es sein. Und daher verspreche ich mir einen großen Erfolg von dem Buch, wenn für derartige Lektüre z. Z.[?] überhaupt dazu gern[?] Bereitschaft vorliegt. Manche meinen nämlich, von all' diesen Dingen, von den tiefsten Tiefen und dem entsetzlichen Grauen der Hitler-Zeit will niemand mehr etwas hören und wissen. Ich glaube das nicht, aber ich kann mich irren natürlich. ${ }^{31}$

Von Seydlitz glaubt zudem, dass der Roman im Ausland auf „großes Interesse“ stoßen könnte. Dies auch deshalb, weil es Gerlach „ausgezeichnet gelungen“ sei, die „furchtbaren Hitlersprüche herauszuarbeiten, die in manchen Menschen in so einer völlig verzweifelten Lage lebendig werden“. Zu den von Heinrich Gerlach angefragten „4 Stellen, die sich mit mir befassen“ macht von Seydlitz umfangreiche Anmerkungen, die die von Gerlach geschilderten Episoden mit den wirklichen Abläufen in Stalingrad ins Verhältnis setzen oder aber auf einzelne Details genauer eingehen. So erklärt von Seydlitz ausführlich, ,wie es zur Lagebeurteilung kam“ und warum die „Einigelung der Armee mit 22 Divisionen“ gegen jegliche militärische Erfahrung war. „Ich kann es nun mal nicht anders ausdrücken“, so der

30 Gerlach an von Seydlitz, 20.11.1956. Der nachfolgende Satz deutet an, wie Gerlach die politische Situation einschätzt: „Ich würde mich um jede Wirkung bringen, wenn ich Sie und ihr Handeln“. Hier bricht der Brief leider ab, die nachfolgende Seite ist nicht im Nachlass verzeichnet.

31 Walther von Seydlitz an Heinrich Gerlach, 12.12.1956. In: Nachlass N55: Walther von SeydlitzKurzbach. Bundesarchiv, Militärarchiv Freiburg, Signatur N55/121, Blatt 10-19, hier Blatt 10. 
ehemalige General in seinem Brief an Gerlach, „nur militärische Idioten konnten überhaupt auf eine solche Idee kommen.“32

\section{Der Dokumentarroman Die verratene Armee - ein Bestseller}

Die Vermutung von Walther von Seydlitz, dass der Roman erfolgreich sein wird, bestätigt sich, Heinrich Gerlachs Roman Die verratene Armee wird - wie andere Romane zum Zweiten Weltkrieg - zum Bestseller. Neben Gerlachs Stalingradroman ist es das Thema der Kriegsgefangenschaft, das die Leser in besonderer Weise interessiert. Dies hängt nicht zuletzt mit dem Umstand zusammen, dass sich noch Tausende Gefangene in der Sowjetunion befinden. Zu den Erfolgstexten gehört daher auch ein Roman wie Josef Martin Bauers So weit die Füße tragen (1955). ${ }^{33}$ Der Verlag betont den dokumentarischen Hintergrund des Textes und verweist darauf, dass die Figur des Clemens Ferell in Wirklichkeit existiert und es sich um eine wahre Geschichte handelt. Damals konnte niemand ahnen, dass die Odyssee um Ferell weitgehend erfunden war. ${ }^{34}$ Jahrzehnte später, 2016, ist dann mit einigem Recht eingeschätzt worden, dass der Roman von Bauer „falsche Nachkriegserinnerungen“ in das kollektive Gedächtnis implantiert und geholfen hat, „das Ent-

32 von Seydlitz an Gerlach, 12.12.1956, Blatt 16.

33 Bauer, der 1901 geboren wurde, war im Dritten Reich bereits als Jugendbuchautor prämiert worden und im Zweiten Weltkrieg Verfasser von propagandistischer Kriegsprosa. 1942 erscheinen von ihm Die Kraniche der Nogaia. Tagebuchblätter aus dem Feldzug im Osten, die 1977(!) bei Herbig „,in einer ungekürzten Neuausgabe“ wieder aufgelegt wurden. 1943 kommt im Zentralverlag der NSDAP die als Tatsachenbericht ausgewiesene Geschichte Unterm Edelweiss in der Ukraine. Eine Gebirgs-Division im Kampf gegen Sowjet-Russland heraus. Den Text von 77 Seiten hat Bauer geschrieben, der Band selbst wird im „Auftrage eines Generalkommandos von Oberstltn. Dr. Egid Gehring“ herausgegeben. Das Geleitwort stammt von dem Generalmajor und Divisionskommandanten Hubert Lanz. „Kampf und Sieg im Feldzug gegen Sowjetrußland der Zukunft zu überliefern, ist Sinn und Zweck dieses Buches“, so Lanz, und er schiebt folgende Begründung nach: „Handelt es sich doch um den schwersten, opfervollsten, aber auch glorreichsten aller Feldzüge dieses Krieges. Keine Kriegsgeschichte, sondern inneres Erlebnis soll Gegenstand der Schilderung sein“, Lanz, Hubert. „Geleitwort“. In: Josef Martin Bauer. Unterm Edelweiss in der Ukraine. Eine Gebirgs-Division im Kampf gegen Sowjet-Russland. Hg. Egid Gehring im Auftrag eines Generalkommandos. München: Eher 1943, 5.

34 Siehe das spannende Radio-Feature Dittlmann, Arthur. „So weit die Füße tragen“. Eine Lange Nacht über Dichtung und Wahrheit eines Welterfolges. Deutschlandfunk vom 17.12.2011. https: //www.deutschlandfunk.de/so-weit-die-fuesse-tragen.704.de.html?dram:article_id=85956 (11.01.2021). 
lastungsbedürfnis der Nachkriegsgesellschaft zu stillen“35. Christian Adam bringt das folgendermaßen auf den Punkt:

Ein Buch mit solch einer Botschaft musste zum Credo einer nach Absolution heischenden westdeutschen Nachkriegsgesellschaft werden. So wie die Geschichte hier ausgelegt wurde, wollte sie eine große Zahl der Deutschen gedeutet wissen. ${ }^{36}$

Man kann es auch so sagen: Bauer - und in anderer Weise auch Fritz Wöss mit Hunde, wollt ihr ewig leben (1958) - entwerfen in ihren Romanen das, was man eine „Wunschbiografie“ nennt. Heinrich Gerlach hingegen lehnt solche Retuschen an der Biografie entschieden ab, aber das Datum der Rückkehr aus der Gefangenschaft wird von ihm am 22. April 1956 ebenfalls mit einer Bilanz versehen:

Jubilate! - Heute bin ich 6 Jahre wieder zu Hause. 6 lange Jahre! Die Bilanz dieser Jahre ist nicht schlecht $u$. es gibt Anlaß, der Fügung sehr dankbar zu sein. Wieder im Beruf, die Familie gesichert, das Buch fertig, ein eigenes Haus ... der Wunsch, noch ein paar Jahre in Ruhe im Kreise der Meinen verbringen zu können - dieser so oft ausgesprochene $u$. noch öfter gedachte heiße Wunsch - ist erfüllt worden. Dank, Dank! ${ }^{17}$

Was Heinrich Gerlach hier notiert, das ist etwas, was in dieser Weise von zahlreichen Kriegsheimkehrern reflektiert wurde, nur oftmals mit dem Unterschied, dass die Bilanz nicht so zufriedenstellend ausfiel wie bei Heinrich Gerlach. Wobei er zu diesem Zeitpunkt noch nicht ahnen konnte, dass seine Verratene Armee ein großer Erfolg werden würde. Mit der 1962 im Spiegel veröffentlichten Liste „Bestseller in der Bundesrepublik“ kann Gerlachs Roman allerdings nicht konkurrieren. Die Liste im Spiegel, die sich an Auflagenhöhen orientiert, kann durchaus als eine Art Spiegel des Bewusstseins eines größeren Teils der damaligen Bevölkerung in der Bundesrepublik gelesen werden. Josef Martin Bauers So weit die Füße tragen liegt mit 780.000 verkauften Exemplaren auf Platz 4, Hans Hellmut Kirst mit dem ersten Teil von 08/15 auf Platz 12 (450.000), der zweite Teil kommt mit 341.500 noch

35 Siehe Fischer, S. „Der Schnee von gestern. Falsche Nachkriegserinnerungen“. In: Süddeutsche Zeitung vom 24.03.2010. https://www.sueddeutsche.de/kultur/falsche-nachkriegserinnerungender-schnee-von-gestern-1.12263 (20.07.2020). Als 2001 die Anonymität des hinter der Geschichte stehenden Cornelius Rost (1919-1983) aufgehoben wird, stellt sich heraus, dass dieser bereits am 28. Oktober 1947 aus sowjetischer Kriegsgefangenschaft zurückgekehrt ist, zu einem Zeitpunkt also, als er eigentlich noch in einem sibirischen Bergwerk in einem Arbeitsbataillon hätte sein sollen.

36 Adam, Christian. Der Traum vom Jahre Null. Autoren, Bestseller, Leser: Die Neuordnung der Bücherwelt in Ost und West nach 1945. Berlin: Galiani 2016, 150.

37 Gerlach, Heinrich. Tagebuch vom 22. April 1956 (Auslassungszeichen i. O., Heinrich Gerlachs Tagebücher sind unveröffentlicht. Eine Auswahl ist in Vorbereitung). 
auf den 18. Platz und der dritte Teil mit immerhin 278.500 auf Platz 23. Auf Platz 14 landet ein weiterer Roman über Stalingrad, nämlich Heinz G. Konsaliks Der Arzt von Stalingrad, der im gleichen Jahr wie Heinrich Gerlachs Roman erscheint. Konsalik verkauft bis 1962415.000 Exemplare. ${ }^{38}$ Es ist kein Zufall, dass die drei Bände der Landser-Romane von Hans Hellmut Kirsts Null-acht-fünfzehn. Die abenteuerliche Revolte des Gefreiten Asch (1954) zu den meistverkauften Büchern zählten. ${ }^{39}$ Kirst wird 1914 in Osterode geboren, jener Stadt, an deren Heeresfachschule der zwei Jahre ältere Heinrich Gerlach 1933 nach dem zweiten pädagogischen Staatsexamen eine erste Anstellung findet. Anders als Gerlach, der Lehrer wurde, entscheidet sich Kirst 1933, die Laufbahn eines Berufsoffiziers einzuschlagen, und ist später in Königsberg, Gerlachs Geburtsstadt, stationiert. Im Zweiten Weltkrieg nimmt er im September 1939 am Einmarsch in Polen teil, ist 1940 - wie Gerlach am Frankreichfeldzug beteiligt und 1941 am Überfall auf die Sowjetunion. Von 1943 bis 1945 wird er Lehrer für Kriegsgeschichte an der Luftkriegsschule im bayerischen Schongau, einige Wochen ist er als Nationalsozialistischer Führungsoffizier eingesetzt. Der Titel des Romans 08/15, zu dem Kirst nach seiner Entlassung aus einem amerikanischen Internierungslager erste Notizen macht, ist eigentlich auf ein deutsches Maschinengewehr zurückzuführen, das seit dem Ersten Weltkrieg eine weite Verbreitung fand und von daher zu einer Redewendung wurde, wenn es um die Bezeichnung von etwas Gewöhnlichem und ständig Wiederkehrendem geht. Genau davon erzählt der Roman, der 1954 erschien, nämlich vom Alltag des Kasernendrills, den Schikanen und kleinen Quälereien, mit denen es der Gefreite Asch zu tun bekommt. ${ }^{40}$ Bereits im Erscheinungsjahr kam der erste Teil einer auf drei Folgen angelegten Verfilmung in die Kinos, mit dem jungen Joachim Fuchsberger in der Hauptrolle. Schon ein Jahr später, 1955, folgten die beiden nächsten Teile. Heinrich Gerlach, der bis in die 1960er Jahre ein großer Kinogänger ist, gibt in seinen Tagebüchern eine kurze Einschätzung der Filmtrilogie. Am 16. April 1956 sieht er den 3. Teil im Kino und notiert im Tagebuch eine

38 Vgl. o. V. Was wird gelesen?. In: Der Spiegel vom 18.04.1962. https://www.spiegel.de/spiegel/ print/d-45139777.html (20.07.2020).

39 Die Neue Illustrierte hatte ab Januar 1954 mit dem Vorabdruck des Romans begonnen, was mit ein Grund für den sich dann einstellenden Erfolg war.

40 Um Hans Hellmut Kirst und seinen Roman 08/15 gab es in der jungen Bundesrepublik schärfste Auseinandersetzungen. Kirst kam auf Grund von Anschuldigungen des späteren Ministerpräsidenten Franz Josef Strauß für neun Monate im amerikanischen Internierungslager in Garmisch in Untersuchungshaft, wurde dann aber als „unbelastet“ entlassen. Auf Grund seiner Kriegserfahrungen lehnte Kirst die Wiederbewaffnung der Bundeswehr entschieden ab. Als der Roman 08/15 1954 erschien, sah Verteidigungsminister Strauß ihn als Affront gegen den Aufbau der Bundeswehr und griff Kirst scharf an. Daraufhin war der Roman in zahlreichen Buchhandlungen in Bayern auf den Index gekommen. 
gleichermaßen präzise wie kritische Wertung: „Heute waren wir alle Mann im Kino zu 08/15 3. Teil. Man wurde genötigt, über Dinge zu lachen, über die niemals gelacht werden dürfte, und deshalb war der Film schlecht. " ${ }^{41}$ Gerlachs Einschätzung trifft sich mit einer aktuellen Sicht von Christan Adam, der darauf verweist, warum Roman wie Film so erfolgreich waren. In einer neuen Untersuchung zur „Neuordnung der Bücherwelt“ nach 1945 gibt er folgende Begründung:

Das Buch zum Maschinengewehr war so erfolgreich, weil es auf harmlose Weise die Frontund Soldatenerzählungen wiederholte, an die sich alle Kriegsteilnehmer noch am ehesten erinnern mochten. Schleifereien und Landser-Humor standen im Vordergrund. Es konnte ein im Gegensatz zum tatsächlichen Vernichtungskrieg harmloses Bild entstehen. Der Text erschien in einer Phase, als die Wiederbewaffnung Westdeutschlands unmittelbar bevorstand..$^{42}$

In der Tat lässt sich sagen, dass Kirst Kriegsbücher schrieb, ,in denen der Krieg nicht vorkam. Erinnerungsliteratur, die die Vergangenheit auf Distanz hielt“. ${ }^{43}$

Fragt man entsprechend danach, welche Rolle die literarischen Texte in der noch jungen Bundesrepublik spielten, so lässt sich sagen, dass sie in spezifischer Weise das hegemoniale Kollektivgedächtnis prägten. Insofern erfüllte Literatur eine stabilisierende Funktion und zielte darauf, durch das Erinnerte eine Stärkung der Gemeinschaft herzustellen. Dabei funktionierte ein Prozess, der darauf hinauslief, für das Kollektivgedächtnis Widersprüche einzuebnen oder aber zu minorisieren und an den Rand abzudrängen. Dies ist erinnerungstheoretisch nur folgerichtig, denn es ging darum, historische Differenzen, Verwerfungen, ja Traumata zugunsten einer konsensfähigen Geschichte im „Dienste nationaler Identitätsstiftung“ aufzubauen. Das Kollektivgedächtnis, mithin die es tragenden Instanzen bzw. Gruppen zielen nämlich darauf - so die Historiker Konrad Jarausch und Martin Sabrow -, „Große Geschichten“ bzw. ,Meistererzählungen' zu installieren. Beim ,soldatischen Opfernarrativ‘, das in zahlreichen Romanen der 1950er Jahre eine zentrale Rolle spielt, ${ }^{44}$ handelt es sich um ei-

41 Gerlach, Heinrich. Tagebuch vom 16. April 1956. Werner Hess, der Filmbeauftragte der Evangelischen Kirche, schätzte ein, dass die Filmemacher wohl eine „scharfe Kritik am Kasernengeist“ beabsichtigt hätten, aber das Gegenteil der Wirkung eingetreten sei: „Die Leute aber, die sich brüllend im Theater auf die Schenkel klatschten und ihrer Eheliebsten in die Seite knufften, stöhnten vor Vergnügen und ächzten freudig: ,Genau so war es, großartig“ “ Hess, Werner. „Filmgeschäft mit dem Krieg“. In: Kirche und Film 7 (1958), 2-5, hier 4.

42 Adam: Der Traum vom Jahre Null, 140.

43 Adam: Der Traum vom Jahre Null, 141.

44 Siehe dazu die profunde Arbeit von Ächtler, Norman. Generation in Kesseln. Das Soldatische Opfernarrativ im westdeutschen Kriegsroman 1945-1960. Göttingen: Wallstein 2013. Vgl. auch Gansel: Nach 70 Jahren aus der Kriegsgefangenschaft zurück, 552 - 571. 
ne solche „kohärente, mit einer eindeutigen Perspektive ausgestattete [...] Geschichtsdarstellung“, deren prägende Kraft nicht nur „innerfachlich schulbildend“ wirkte, sondern „öffentliche Dominanz“ erfuhr. Die ,Meistererzählung“ vom Soldaten als Leidtragendem und Opfer gewann erst „durch ihre Materialisierung, Verbreitung und Institutionalisierung“ eine gesamtgesellschaftliche Geltung in der Bundesrepublik. Dass sich also das ,soldatische Opfernarrativ seit Ende der 1940er Jahre durchsetzte, hing damit zusammen, dass es „kulturelle Zeitströmungen“ reflektierte, „den Ton der Zeit“ traf und über „geeignete Mittel und Wege“ verfügte, sich „fachwissenschaftlich und außerfachlich Gehör zu verschaffen.“ 45

Betrachtet man vor diesem Hintergrund Heinrich Gerlachs Stalingradroman Die verratene Armee, dann erklärt sich, warum er erfolgreich war, obwohl er mit der Darstellung der Schrecken keineswegs ein einseitiges Entlastungsnarrativ präsentierte. In der Neukonstruktion des Romans ab dem Beginn der 1950er Jahre spielen - anders als in der Urfassung von 1945 - das ,soldatische Opfernarrativ‘, eschatologisch aufgeladene christliche Untergangsszenarien wie auch das Motiv der Wandlung eine wichtige Rolle. Das Schicksal der 6. Armee steht sinnbildhaft für die Zerstörung der noch erhaltenen Reste an Zivilisation. Der Erzähler schildert, wie die Hauptfigur in einem symbolischen Akt Kennzeichen des bisherigen Daseins zu verbrennen sucht:

Breuer schleppt seinen Koffer selbst zu dem Scheiterhaufen. [...] Bücher, Zeitungen, Briefe und Bilder... Stück für Stück wirft er ins Feuer und mit jedem verbrennt ein Stück Vergangenheit. [...] Eingeschlossen, aus! Der Lebensfaden nach drüben ist abgeschnitten. ${ }^{46}$

Und später wird Breuer, wie Heinrich Gerlach dies in der Realität auch getan hat, sein Tagebuch verbrennen. Da, wo es keine Perspektive gibt, bedarf es auch keiner Erinnerung an das Vergangene:

Breuer gab sich einen Ruck und warf das Buch [Tagebuch] in die Flammen.... und schaute nicht zurück! Es gab keine Zukunft mehr und also auch keine Vergangenheit. Es gab nur noch das Hier und das Jetzt, wie Unold gesagt hatte.

Doch dann lag ihm das Päckchen Briefe in der Hand. Die Briefe von seiner Frau, von Irmgard. Erst wenn sie alle dort in der Glut verkohlten, würde der wirklich bereit sein. ${ }^{47}$

45 Jarausch, Konrad H./Sabrow, Martin. „,Meistererzählung“ - Zur Karriere eines Begriffs“. In: Die historische Meistererzählung: Deutungslinien der deutschen Nationalgeschichte nach 1945. Hg. Konrad H. Jarausch, Martin Sabrow. Göttingen: V\&R 2002, 9-32, hier 16, 17, 21.

46 Gerlach: Die verratene Armee, 86.

47 Gerlach: Die verratene Armee, 318. 
Dort, wo Heinrich Gerlach die deutschen Soldaten als Opfer von Gewalt und Krieg erfasst, bietet der Roman also durchaus ein Identifikationsangebot für die heimkehrende Erfahrungs- und Erinnerungsgemeinschaft der Frontsoldaten. ${ }^{48}$ Gleichzeitig aber liegt Gerlachs Stalingradroman mit der Darstellung der Schrecken des Krieges quer zu den in der Literatur überwiegenden Entlastungsnarrativen. Dass der Roman dennoch seine Leser fand, hängt damit zusammen, dass auch in der bundesdeutschen Nachkriegsgesellschaft unterschiedliche, ja gegensätzliche Erinnerungskulturen miteinander im Widerstreit lagen und sich durchmischten. Dies ist ein gänzlich normaler Vorgang. Schon vor dem Zweiten Weltkrieg hatte Walter Benjamin in seinen viel zitierten Geschichtsphilosophischen Thesen darauf aufmerksam gemacht, dass die Geschichte „Gegenstand einer Konstruktion“ ist und es sich um eine mit „Jetztzeit geladene Vergangenheit“ handelt. ${ }^{49}$ Und der französische Soziologe und Philosoph Maurice Halbwachs vermutet in seiner Theorie vom kollektiven Gedächtnis, dass

\begin{abstract}
wir in der Erinnerung von der Gegenwart, vom System der allgemeinen Ideen aus[gehen], das uns immer zur Verfügung steht [...], wir kombinieren sie, um entweder ein bestimmtes Detail oder eine Nuance vergangener Gesichter oder Ereignisse und allgemein unserer früheren Bewusstseinszustände wiederzufinden. ${ }^{50}$
\end{abstract}

Diese Rekonstruktion, so Halbwachs, würde aber „nur annäherungsweise“ gelingen, wenn es „eine Lücke im Eindruck [gibt], die den Anpassungsmangel des sozialen Verständnisses an die Bedingungen unseres persönlichen früheren Bewusstseinslebens misst. "51 Geht man - hier nur sehr verkürzt - von diesen Überlegungen aus, dann kann man die damaligen „Tricks der Erinnerung“ (Uwe John-

48 In der im Gefangenenlager geschriebenen Urfassung Durchbruch bei Stalingrad sind die Momente des Untergangs, der Opferung wie auch der Wandlung weitaus zurückhaltender fixiert. In der nach der Rückkehr zwischen 1951 und 1956 neu geschriebenen zweiten Variante liefert der im Kessel erfahrene Verlust alles Menschlichen die Voraussetzung, um den neuen Weg ,in Demut zu gehen“ (457).

49 Benjamin, Walter. „Über den Begriff der Geschichte“. In: Walter Benjamin. Gesammelte Schriften. Werkausgabe. Band I.2. Abhandlungen. Hg. Rolf Tiedemann, Hermann Schweppenhäuser. Frankfurt a. M.: Suhrkamp 1974, 691-704, hier 701.

50 Halbwachs, Maurice. Das kollektive Gedächtnis. Frankfurt a. M.: Fischer 1985, 55.

51 Halbwachs: Das kollektive Gedächtnis, 55. Siehe dazu Gansel, Carsten (Hg.). Gedächtnis und Literatur in den ,geschlossenen Gesellschaften ‘ des Real-Sozialismus zwischen 1945 und 1989. Göttingen: V\&R 2007. Vgl. auch die Ergebnisse des Gießener Sonderforschungsbereiches 434 „Erinnerungskulturen“ in der von Jürgen Reulecke und Birgit Neumann herausgegebenen Reihe ,Formen der Erinnerung'. Siehe in diesem Rahmen u. a. auch: Erll, Astrid/Nünning, Ansgar (Hg.). Medien des kollektiven Gedächtnisses. Konstruktivität, Historizität, Kulturspezifizität. Berlin/New York: De Gruyter 2004. 
son) ${ }^{52}$ durchaus kulturgeschichtlich verorten. Martin Sabrow hat entsprechend die leitenden Topoi der westdeutschen Erinnerung im öffentlichen Bewusstsein der ersten beiden Jahrzehnte wie folgt zusammengefasst:

\begin{abstract}
Im Vordergrund standen das Grauen des Bombenkriegs und die Zerstörung der Städte, die Umstände von Flucht und Vertreibung aus den Ostgebieten und das Schicksal der Soldaten in der Kriegsgefangenschaft. Öffentliche Aufmerksamkeit wurde daneben, wenngleich zögernd und heftig umkämpft, dem militärischen und dem christlichen Widerstand gegen das NS-Regime zuteil, während dessen Terrormaschinerie und die in den Holocaust mündende Verfolgung von Juden und anderen als ,fremdrassig‘ aus der Volksgemeinschaft Ausgegrenzten lange Zeit nur einen randständigen Platz in der öffentlichen Erinnerung fanden. ${ }^{53}$
\end{abstract}

Ende der 1950er Jahre wurde das hegemoniale Kollektivgedächtnis zunehmend aufgebrochen, und an die Stelle von Entlastung trat eine kritische Auseinandersetzung mit den Jahren von 1933 bis 1945, die versuchte, das Verhältnis von Tätern und Opfern in den Blick zu bekommen. Unter diesen Bedingungen konnte Heinrich Gerlach auch darangehen, seinen zweiten Dokumentarroman Odyssee in Rot zu beginnen, an dem er neun Jahre arbeitete.

\title{
Literatur
}

Ächtler, Norman. Generation in Kesseln. Das Soldatische Opfernarrativ im westdeutschen Kriegsroman 1945-1960. Göttingen: Wallstein 2013.

Adam, Christian. Der Traum vom Jahre Null. Autoren, Bestseller, Leser: Die Neuordnung der Bücherwelt in Ost und West nach 1945. Berlin: Galiani 2016.

Bauer, Josef Martin. Die Kraniche der Nogaia. Tagebuchblätter aus $d$. Feldzug im Osten. München: Piper 1942.

Bauer, Josef Martin. Unterm Edelweiss in der Ukraine. Eine Gebirgs-Division im Kampfgegen Sowjet-Russland. Hg. Egid Gehring im Auftrag eines Generalkommandos. München: Eher 1943.

Bauer, Josef Martin. Die Kraniche der Nogaia. Ukrainisches Tagebuch. Ungekürzte Neuausgabe. München/Berlin: Herbig 1977.

Benjamin, Walter. „Über den Begriff der Geschichte“. In: Walter Benjamin. Gesammelte Schriften. Werkausgabe. Band I.2. Abhandlungen. Hg. Rolf Tiedemann, Hermann Schweppenhäuser. Frankfurt a. M.: Suhrkamp 1974, 691-704.

52 Johnson, Uwe. „Versuch, eine Mentalität zu erklären. Über eine Art DDR-Bürger in der Bundesrepublik Deutschland“. In: Uwe Johnson. Berliner Sachen. Frankfurt a. M.: Suhrkamp 1975, 52-63, hier 63.

53 Sabrow, Martin. „Den Zweiten Weltkrieg erinnern“. In: Aus Politik und Zeitgeschichte 36-37 (2009), 14-21, hier 15-16. 
Bidmon, Agnes. „Dokufiktion - Neue Formen realistischen Erzählens in der deutschsprachigen Gegenwartsliteratur“. In: Dokumentarisches Erzählen - Erzählen mit Dokumenten in Literatur, Theater und Film. Hg. Carsten Gansel, Peter Braun. Berlin: Okapi Verlag 2020, 103-130.

Dittlmann, Arthur. „So weit die Füße tragen“. Eine Lange Nacht über Dichtung und Wahrheit eines Welterfolges. Deutschlandfunk vom 17.12.2011. http://www.deutschlandfunk.de/soweit-die-fuesse-tragen.704.de.html (05.09.2016).

Erll, Astrid/Nünning, Ansgar (Hg.). Medien des kollektiven Gedächtnisses. Konstruktivität, Historizität, Kulturspezifizität. Berlin/New York: De Gruyter 2004.

Fischer, S. „Der Schnee von gestern. Falsche Nachkriegserinnerungen“. In: Süddeutsche Zeitung vom 24.03.2010. https://www.sueddeutsche.de/kultur/falschenachkriegserinnerungen-der-schnee-von-gestern-1.12263 (20.07.2020).

Gansel, Carsten (Hg.). Gedächtnis und Literatur in den, geschlossenen Gesellschaften ' des Real-Sozialismus zwischen 1945 und 1989. Göttingen: V\&R 2007.

Gansel, Carsten. „Nach 70 Jahren aus der Kriegsgefangenschaft zurück - Heinrich Gerlachs Roman Durchbruch bei Stalingrad und seine abenteuerliche Geschichte“. In: Heinrich Gerlach. Durchbruch bei Stalingrad. Hg. Carsten Gansel. Berlin: Galiani 2016, 519-693.

Gansel, Carsten. „Widerstandsheld, Vaterlandsverräter, wacher Demokrat und Zeitzeuge? - Heinrich Gerlach, seine Odyssee durch die sowjetischen Gefangenenlager und sein Schicksal in der sich neu formierenden Bundesrepublik“. In: Heinrich Gerlach. Odyssee in Rot. Bericht einer Irrfahrt. Hg. Carsten Gansel. Berlin: Galiani 2017, 691-915.

Gansel, Carsten. „Ursula Krechels Landgericht (2012) und die ,verlorenen Erinnerungen“ der Nachkriegszeit oder Erinnerungskulturen zwischen Verdrängen und Vergessen“. In: Deutschunterricht (6/2019), 32-43.

Gerlach, Heinrich. „Wendepunkt Stalingrad. Rückzug nach Osten“. In: Freies Deutschland vom 21.11.1943, 1.

Gerlach, Heinrich. „Der ,Geist von Stalingrad““. In: Freies Deutschland vom 26.03.1944, 1.

Gerlach, Heinrich. „2 Jahre nach Stalingrad. Der neue Weg“. In: Freies Deutschland vom 04.02.1945, 1.

Gerlach, Heinrich. Tagebuch. Braake 1956. Unv.

Gerlach, Heinrich. Die verratene Armee. München: Verlagshandlung 1957.

Gerlach, Heinrich. Die verratene Armee. Ein Stalingrad-Roman. 2., bearbeitete Taschenbuchausgabe. München: Wilhelm Heyne 1976.

Gerlach, Heinrich. „Nachwort“. In: Heinrich Gerlach. Die verratene Armee. Ein StalingradRoman. 2., bearbeitete Taschenbuchausgabe. München: Wilhelm Heyne 1976, 309-311.

Gerlach, Heinrich. Durchbruch bei Stalingrad. Hg. Carsten Gansel. Berlin: Galiani 2016.

Gerlach, Heinrich. Doorbraak bij Stalingrad. Hg. Carsten Gansel. Amsterdam: Uitgegeven door Xander Uitgevers BV 2017.

Gerlach, Heinrich. Éclairs lointains Percée Stalingrad. Hg. Carsten Gansel. Paris: Éditions Anne Carrière 2017.

Gerlach, Heinrich. Odyssee in Rot. Bericht einer Irrfahrt. Berlin: Galiani 2017.

Gerlach, Heinrich. Breakout at Stalingrad. Hg. Carsten Gansel. London: Apollo/Head of Zeus Ltd. 2018.

Gerlach, Heinrich an Walther von Seydlitz, 20.11.1956. In: Nachlass N55: Walther von SeydlitzKurzbach. Bundesarchiv, Militärarchiv Freiburg, Signatur N55/121.

Halbwachs, Maurice. Das kollektive Gedächtnis. Frankfurt a. M.: Fischer 1985.

Hess, Werner. „Filmgeschäft mit dem Krieg“. In: Kirche und Film 7 (1958), 2-5. 
Jarausch, Konrad H./Sabrow, Martin. „,Meistererzählung“ - Zur Karriere eines Begriffs“. In: Die historische Meistererzählung: Deutungslinien der deutschen Nationalgeschichte nach 1945. Hg. Konrad H. Jarausch, Martin Sabrow. Göttingen: V\&R 2002, 9-32.

Johnson, Uwe. „Versuch, eine Mentalität zu erklären. Über eine Art DDR-Bürger in der Bundesrepublik Deutschland“. In: Uwe Johnson. Berliner Sachen. Frankfurt a. M.: Suhrkamp 1975, 52-63.

Kirst, Hans Hellmut. 08/15. Teil 1. 08/15 in der Kaserne [Originaltitel: Die abenteuerliche Revolte des Gefreiten Asch]. Köln: Lingen 1954.

Kirst, Hans Hellmut. 08/15. Teil 2. 08/15 im Krieg [Originaltitel: Die seltsamen Kriegserlebnisse des Soldaten Asch]. Köln: Lingen 1954.

Kirst, Hans Hellmut. 08/15. Teil 3. 08/15 bis zum Ende [Originaltitel: Der gefährliche Endsieg des Soldaten Asch]. Köln: Lingen 1954.

Lanz, Hubert. „Geleitwort“. In: Josef Martin Bauer. Unterm Edelweiss in der Ukraine. Eine Gebirgs-Division im Kampfgegen Sowjet-Russland. Hg. Egid Gehring im Auftrag eines Generalkommandos. München: Eher 1943, 5.

Lejeune, Philippe. Der autobiographische Pakt. Aus dem Französischen von Wolfram Bayer und Dieter Hornig. Frankfurt a. M.: Suhrkamp 1994.

o. V. „Was wird gelesen?“. In: Der Spiegel vom 18.04.1962. https://www.spiegel.de/spiegel/ print/d-45139777.html (20.07.2020).

Petrick, Birgit. ,Freies Deutschland“ - die Zeitung des Nationalkomitees ,Freies Deutschland“ (1943-1945). München: K.G. Saur 1979.

Platthaus, Andreas. „In der Sache Kornitzer“. In: Frankfurter Allgemeine Zeitung vom 10.10.2012.

Sabrow, Martin. „Den Zweiten Weltkrieg erinnern“. In: Aus Politik und Zeitgeschichte 36-37 (2009), 14-21.

Seydlitz, Walther von an Heinrich Gerlach vom 12. Dezember 1956. In: Nachlass N55: Walther von Seydlitz-Kurzbach. Bundesarchiv, Militärarchiv Freiburg, Signatur N55/121, Blatt $10-19$.

Seydlitz, General Walther von. „Die Beurteilung der Lage der 6. Armee im Kessel von Stalingrad“. In: Martens, Hans. General von Seydlitz 1942-1945. Analyse eines Konflikts. Berlin: edition kloeden 1971, 76-81.

Zipfel, Frank. „Autofiktion. Zwischen Grenzen von Faktualität, Fiktionalität und Literarizität“. In: Revisionen. Grundbegriffe der Literaturtheorie. Hg. Fotis Jannidis u. a. Berlin/New York: De Gruyter 2009, 285-314. 